\title{
The acute surgical unit as a novel model of care for patients presenting with acute cholecystitis
}

Lester Pepingco BMedSc, MB BS(Hons) Resident Medical Officer

Guy D Eslick PhD, FACE, FFPH, Associate Professor of Surgery and Cancer Epidemiology ${ }^{2}$

Michael R Cox MB BS, FRACS, MS Professor of Surgery

1 Royal Prince Alfred Hospital, Sydney, NSW. 2 Department of Surgery, The University of Sydney. Sydney, NSW.

guy.eslick@ sydney.edu.au

MJA2012; 196: 509-511 doi: 10.5694/mjall.1136 cute cholecystitis secondary to gallstones is a common acute general surgical presentation. During early experience with laparoscopic cholecystectomy, acute cholecystitis was considered a contraindication. ${ }^{1}$ However, several studies showed that early cholecystectomy was safe. ${ }^{2-6}$ More recently, randomised controlled trials and a Cochrane review have shown that early cholecystectomy results in lower rates of morbidity, similar rates of conversion to open surgery, reduced hospital stay and lower economic cost when compared with delayed cholecystectomy. $^{7-11}$

An acute surgical unit (ASU) was adopted by Nepean Hospital (a teaching hospital) in November 2006. The ASU is a novel consultant-led model of care for assessing and treating all patients who present with an acute general surgical condition. ${ }^{12}$ The ASU team consists of a consultant surgeon, two surgical registrars, two resident medical officers and a nurse practitioner working on a 12-hour shift ( $7 \mathrm{am}$ to $7 \mathrm{pm}$ ). The consultant's sole commitment during a shift is management of patients in the ASU. The team functions in the same way every day of the year, including weekends and public holidays. Overnight, there is a dedicated ASU registrar in the hospital and the consultant is on call. All patients who present with acute general surgical conditions or trauma are admitted into and stay under the care of the ASU. Patients with common conditions are managed according to agreed evidence-based bestpractice protocols.

The ASU team was set up at Nepean Hospital to enable more rapid assessment of emergency patients, more timely operative procedures and a more efficient use of

Objective: To determine whether the introduction of an acute surgical unit (ASU) resulted in a greater proportion of patients with acute cholecystitis receiving definitive surgery on index admission with no adverse change in surgical outcomes.

Design, setting and participants: A retrospective study of medical records for patients presenting to Nepean Hospital with acute cholecystitis during the 2 years before and 2 years after introduction of an ASU in November 2006.

Main outcome measures: Time to diagnosis, timing of surgical intervention, surgical outcomes, duration of total admission and complication rates.

Results: A total 271 patients were included in the study (114 pre-ASU, 157 postASU). After introduction of the ASU, a higher proportion of patients had surgery on index admission ( $89.8 \% \vee 55.3 \% ; P<0.001)$ and there were decreases in median time to diagnosis ( $14.9 \mathrm{~h} \vee 10.8 \mathrm{~h} ; P=0.008$ ), median time to definitive procedure ( 5.6 days $\vee 2.1$ days; $P<0.001$ ), median duration of total admission ( 4.9 days $\vee 4.0$ days; $P=0.002$ ), rate of intraoperative conversion to open surgery $(14.9 \% \vee 4.5 \% ; P=0.003)$ and rate of postoperative infection $(3.5 \% \mathrm{v}$ $2.5 \% ; P=0.40)$.

Conclusion: Introduction of the ASU at Nepean Hospital resulted in significant improvements in care and outcomes for patients with acute cholecystitis.

operating theatres. Before November 2006, Nepean Hospital had a traditional on-call system (where the patient was admitted under the consultant for the day and managed according to that surgeon's opinion) and had no consensus on management of patients with acute cholecystitis. The ASU protocol for patients with acute cholecystitis was laparoscopic cholecystectomy on the index admission.

We aimed to determine whether the introduction of the ASU resulted in improvements in care and outcomes for patients with acute cholecystitis.

\section{Methods}

A retrospective study of medical records for patients presenting to Nepean Hospital with acute cholecystitis was conducted over a 4-year period (1 November 2004 to 30 October 2008) - the 2 years before and 2 years after introduction of the ASU
(Box 1). The study was approved by the Sydney West Area Health Service Ethics Committee.

Patients were included if they presented with a history and clinical signs consistent with a diagnosis of acute cholecystitis, confirmed by ultrasound findings of gallstones and associated ultrasound signs of gallbladder inflammation. Patients with biliary tree obstruction (jaundice, cholangitis or biliary pancreatitis) were not included.

Two linked databases were set up to de-identify the data. Data were collected on age, sex, date and time of presentation to the emergency department, date and time of assessment by the surgical team, date and time of diagnosis, whether surgery was performed on index admission, date of discharge from hospital, whether surgery commenced during daylight hours (defined as 8 am to $5 \mathrm{pm}$ ), intraoperative conversion to open surgery, and postoperative complications. 
Data were analysed using Prism version 5.03 for Windows (GraphPad Software, San Diego, Calif, USA) and the Mann-Whitney $U$ test. A $P$ value of $<0.05$ was considered significant.

\section{Results}

A total of 271 consecutive patients met the inclusion criteria; 114 presented before the ASU was introduced and 157 presented after. The key results are summarised in Box 2 . The median age was 44.4 years (range, 14-85 years) in the pre-ASU group and 45.1 years (range, 14-89 years) in the post-ASU group ( $P=$ 0.01 ) and there were more females in both groups.

Before the introduction of the ASU, the proportion of patients who had surgery on index admission was significantly lower (55.3\% v 89.8\%) and the proportion of patients for whom surgery commenced during daylight hours was significantly lower (82.5\% v 91.7\%).

After the introduction of the ASU, there were significant decreases in the median time to diagnosis (14.9 hours v 10.8 hours), median time to definitive procedure (5.6 days v 2.1 days) and median duration of total admission (4.9 days $\mathrm{v} 4.0$ days). There was also was a significant reduction in the rate of intraoperative conversion from laparoscopic to open surgery $(14.9 \% \mathrm{v} 4.5 \%)$. No significant changes in the rates of postoperative complications were found.

\section{Discussion}

Introduction of the ASU model of care at Nepean Hospital meant that most patients who presented with acute cholecystitis had surgery on index admission, compared with just over half of those who presented before the model was introduced.

The ASU model also resulted in more rapid diagnosis and earlier surgery (3 days earlier in the admission on average). Although more patients had definitive surgery on index admission, the overall hospital stay was almost a day shorter. These improved efficiencies are due to the model being consultant-led and having a dedicated team available throughout the day to assess and treat patients. Another improvement in patient care was the increase in the proportion of patients who had surgery during daylight hours. Recent studies have highlighted that operating outside of daylight hours is associated with impairments in speed, accuracy and dexterity that contribute to potentially life-threatening errors. $^{13-15}$

A significant reduction in the conversion rate from laparoscopic to open surgery was also shown. This is likely to result in patients returning to normal activities more rapidly, although we did not assess this. A possible reason for the reduced conversion rate under the ASU model is that surgery is done on patients who have a less severely inflamed gallbladder. This could be due to surgery being performed sooner during the index admission - before the development of severe inflammation that results in conversion. Furthermore, during the pre-ASU period, patients with less severe inflammation that resolved with non-operative treatment were discharged rather than having surgery performed during the index admission.

Introduction of the ASU at Nepean Hospital resulted in significant improvements in patient outcomes and hospital efficiencies relating to acute cholecystitis. Our results support the development of new models of care for surgical patients and offer significant advantages for patients and the health care system.

Competing interests: No relevant disclosures.

Received 25 Oct 2011, accepted 11 Apr 2012.
1 Tompkins RK. Laparoscopic cholecystectomy: threat or opportunity? Arch Surg 1990; 125: 1245.

2 Cox M, Wilson T, Luck A, et al. Laparoscopic cholecystectomy for acute inflammation of the gallbladder. Ann Surg 1993; 218: 630-634.

3 Jacobs M, Verdeja JC, Goldstein HS. Laparoscopic cholecystectomy in acute cholecystitis. J Laparoendosc Surg 1991; 1: 175-177.

4 Cuschieri A, Dubois F, Mouiel J. The European experience with laparoscopic cholecystectomy. Am J Surg 1991; 161: 385-387.

5 Peters JH, Gibbons GD, Innes JT, et al. Complications of laparoscopic cholecystectomy. Surgery 1991; 110: 769-778.

6 Peters JH, Ellison EC, Innes JT, et al. Safety and efficacy of laparoscopic cholecystectomy. A prospective analysis of 100 initial patients. Ann Surg 1991; 213: 3-12.

7 Keus F, de Jong JA, Gooszen HG, van Laarhoven CJ. Laparoscopic versus open cholecystectomy for patients with symptomatic cholecystolithiasis. Cochrane Database Syst Rev 2006; (4): CD006231.

8 Lawrentschuk N. Hewitt P. Pritchard M. Elective laparoscopic cholecystectomy: implications of prolonged waiting times for surgery. ANZ J Surg 2003; 73: 890-893.

9 Sobolev B, Mercer D, Brown P, et al. Risk of emergency admission while awaiting elective cholecystectomy. CMAJ 2003; 169: 662-665.

10 Lai P, Kwong K, Leung K, et al. Randomized trial of early versus delayed laparoscopic cholecystectomy for acute cholecystitis. Br J Surg 1998; 85: 764-767.

11 Lo C, Liu C, Fan S, Lai E. Prospective randomised study of early versus delayed laparoscopic cholecystectomy for acute cholecystitis. Ann Surg 1998; 227: 461-467.

12 Cox M, Cook L, Dobson J, et al. Acute surgical unit: a new model of care. ANZ J Surg 2010; 80: 419-424.

13 Grantcharov TP, Bardram L, Funch-Jensen P, Rosenberg J. Laparoscopic performance after one night on call in surgical department: prospective study. BMJ 2001; 323: 1222-1223.

14 Taffinder N, McManus I, Gul Y, et al. Effect of sleep deprivation on surgeons' dexterity on laparoscopy simulator. Lancet 1998; 352: 1191.

15 Ricci WM, Gallagher B, Brandt A, et al. Is after-hours orthopaedic surgery associated with adverse outcomes? J Bone Joint Surg Am 2009; 91: 2067-2072.

1 Management of patients with acute cholecystitis before and after introduction of an acute surgical unit (ASU) at Nepean Hospital

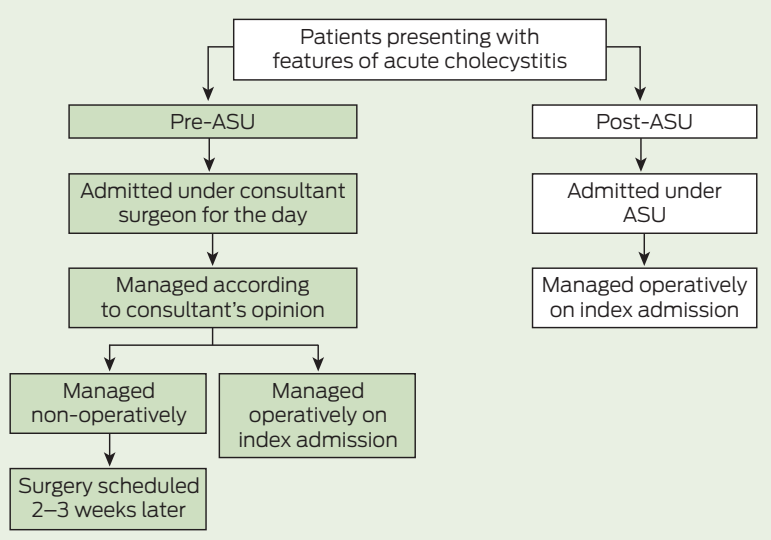

\section{Demographics, surgical outcomes and complications for patients who presented to Nepean Hospital with acute cholecystitis before and after introduction of an ASU*}

\begin{tabular}{|c|c|c|c|}
\hline & Pre-ASU $(n=114)$ & Post-ASU $(n=157)$ & $P$ \\
\hline Median age (years) & 44.4 & 45.1 & 0.01 \\
\hline Females & $82(71.9 \%)$ & $96(61.1 \%)$ & 0.04 \\
\hline Surgery performed on index admission & $63(55.3 \%)$ & $141(89.8 \%)$ & $<0.001$ \\
\hline Surgery commenced during daylight hours & $94(82.5 \%)$ & $144(91.7 \%)$ & 0.013 \\
\hline Median time to diagnosis (hours) & 14.9 & 10.8 & 0.008 \\
\hline Median time to definitive procedure (days) & 5.6 & 2.1 & $<0.001$ \\
\hline Median duration of total admission (days) & 4.9 & 4.0 & 0.002 \\
\hline Intraoperative conversion from laparoscopic to open surgery & $17(14.9 \%)$ & $7(4.5 \%)$ & 0.003 \\
\hline Postoperative deep venous thrombosis & 0 & 0 & - \\
\hline Postoperative infection & $4(3.5 \%)$ & $4(2.5 \%)$ & 0.40 \\
\hline Bile duct injury & 0 & 0 & - \\
\hline Postoperative jaundice & $1(0.9 \%)$ & 0 & 0.10 \\
\hline
\end{tabular}

ASU = acute surgical unit. * Data are number (\%) unless otherwise indicated. 
Research 